\title{
Plasma rotation in a quasisymmetric stellarator
}

\author{
Andrei N. Simakov ${ }^{1,2}$ and Per Helander ${ }^{2}$ \\ ${ }^{1}$ Theoretical Division, Los Alamos National Laboratory, \\ Los Alamos, New Mexico 87545, U.S.A. \\ ${ }^{2}$ Max-Planck-Institut für Plasmaphysik, EURATOM-Association, \\ 17491 Greifswald, Germany
}

(June 22, 2010)

\begin{abstract}
The equilibrium plasma rotation in a general toroidal magnetic field is nearly always subsonic and is determined by the requirement that the collisional particle transport should be ambipolar in lowest order in the small-ion-gyroradius expansion. Gyrokinetic turbulence can only appreciably modify the rotation on radial length scales of order the ion gyroradius. Only in quasisymmetric fields, where collisional particle transport is intrinsically ambipolar, can the plasma rotate freely and then only in the quasisymmetry direction. Sonic rotation velocities are allowed in this case. However, the quasisymmetry gets violated when the rotation speed exceeds the diamagnetic speed appreciably, leading to reappearance of the non-ambipolar $1 / \nu$-transport regime. Fortunately, this transport scales with the fourth power of the rotation Mach number and is expected to be modest in most plasmas of interest.
\end{abstract}




\section{INTRODUCTION}

One of the most important lines of stellarator optimization is the quest for quasisymmetric configurations [1-3]. By definition, the strength of a quasisymmetric magnetic field is independent of some linear combination of the Boozer angles. Thus,

if $\theta_{B}$ and $\varphi_{B}$ are the poloidal and toroidal angles in the Boozer coordinates, $\boldsymbol{B}$ is quasisymmetric if $B \equiv|\boldsymbol{B}|$ is independent of $m \theta_{B}-n \varphi_{B}$, where $m$ and $n$ are integers. It is remarkable that a magnetic field can be quasisymmetric (at least approximately) without being symmetric in the usual sense, i.e., that $B$ can be symmetric although $\boldsymbol{B}$ is not [4].

In a perfectly quasisymmetric magnetic field, all guiding-center orbits are well confined and obey Tamm's theorem: they do not depart from a given flux surface by more than a gyroradius multiplied by a geometric constant. The reason is that the guiding-center Lagrangian, when written in the Boozer coordinates, only depends on the magnitude but not on the direction of $\boldsymbol{B}$. A constant of motion therefore exists in addition to the energy and magnetic moment, namely, the canonical angular momentum conjugate to the ignorable angle $m \theta_{B}-n \varphi_{B}$, in analogy to the canonical toroidal angular momentum in an axisymmetric tokamak. In fact, the analogy goes further. All the standard neoclassical properties of tokamaks carry over to quasisymmetric stellarators, because there is an isomorphism between the drift kinetic equations in these configurations [6]. The usual neoclassical results for tokamaks therefore also apply to quasisymmetric stellarators, and the transport coefficients can be translated by a simple recipe. Some of these coefficients can change sign - the bootstrap current in a quasihelical stellarator is normally negative, for instance - but no new equations need to be solved for the stellarator case if the relevant tokamak results are known. (This is actually also true for the much wider class of omnigenous, or quasi- 
isodynamic, stellarators [5].) In particular, the neoclassical transport is benign and lacks the " $1 / \nu$-regime" otherwise plaguing stellarator confinement.

A special feature of the tokamak is that toroidal plasma rotation is almost unrestricted, because the neoclassical transport is intrinsically ambipolar [7, 8]. This means that the radial fluxes of ions and electrons are equal and independent of the radial electric field in leading order in the gyroradius expansion. Because of the isomorphism just mentioned, quasisymmetric stellarators also have this property, and the plasma is free to rotate in the symmetry direction. One can prove that this remains true in a turbulent plasma as long as the fluctuations satisfy the usual gyrokinetic ordering [9]. However, quasisymmetric fields are the only configurations enjoying intrinsic ambipolarity: in all other magnetic fields the rotation is damped to the value required for ambipolar radial transport. The fact that quasisymmetry facilitates rotation has sometimes been emphasized as an advantage, since it can lead to the reduction of turbulent transport [10].

In the present paper, we investigate what happens when a quasisymmetric stellarator plasma is made to rotate rapidly, and find that as soon as the rotation velocity starts to exceed the diamagnetic speed significantly, this leads to a loss of the properties usually associated with quasisymmetry: intrinsic ambipolarity no longer holds and a $1 / \nu$-regime reappears in the neoclassical transport. This happens because, like in the tokamak, the centrifugal force associated with the rotation causes the density and electrostatic potential to vary within each flux surface, but unlike the tokamak, the ensuing electric field is not quasisymmetric. The $\boldsymbol{E} \times \boldsymbol{B}$ drift therefore destroys the perfect confinement of collisionless orbits. Mathematically, what causes a rotating plasma to behave differently in tokamaks and quasisymmetric stellarators is the circumstance that the drift kinetic equation appropriate for describing a rapidly rotating plasma does not exhibit the isomorphism mentioned above for non-rotating 
plasmas.

Specifically, we explicitly evaluate the electron transport in the $1 / \nu$-regime caused by a rapid rotation of a quasisymmetric plasma. Fortunately, this transport turns out to be relatively weak. It is proportional to $M^{4} / \nu_{e}$ multiplied by a small geometric factor, where $\nu_{e}$ is the electron collision frequency and $M$ is the Mach number associated with the rotation speed. In most plasmas of interest $M$ is expected to be small, so the extra transport caused by this mechanism may be modest.

This paper is organized as follows. Section II briefly reviews the topic of a subsonically rotating toroidal plasma and shows that unrestricted rotation is only possible in quasisymmetric configurations [9]. Sections III and IV discuss the case of a rapidly rotating toroidal plasma. In particular, section III briefly reviews the equilibrium rotation of such a plasma [11], and section IV explicitly evaluates the electron transport in the $1 / \nu$-regime caused by the rotation. Finally, section $\mathrm{V}$ summarizes the results and presents conclusions.

\section{A SLOWLY ROTATING TOROIDAL PLASMA}

We begin by briefly reviewing the topic of a subsonically rotating toroidal plasma, as discussed in detail in $[9,12]$. Specifically, we show that collisional plasma transport is intrinsically ambipolar only in a quasisymmetric toroidal magnetic field. Consequently, only in such a field can the plasma rotate freely, and then only in the quasisymmetry direction. In all other magnetic field configurations the rotation is determined by the radial electric field value required for ambipolar collisional particle transport. Moreover, gyrokinetic turbulence is unable to significantly affect this result.

We neglect plasma turbulence at first and observe that the first-order contribution 
(in the small-gyroradius expansion) to the gyrophase-averaged distribution function $f_{j}=f_{j 0}+f_{j 1}+\ldots$ for plasma species $j$ is determined by the standard drift-kinetic equation $[13]$

$$
v_{\|} \nabla_{\|} f_{j 1}+\boldsymbol{v}_{d j} \cdot \nabla f_{j 0}=C_{j}\left(f_{j 1}\right)
$$

Equation (1) employs the total energy $\varepsilon \equiv v^{2} / 2+e_{j} \phi / m_{j}$ and magnetic moment $\mu \equiv v_{\perp}^{2} /(2 B)$ as the independent velocity variables, with $e_{j}$ and $m_{j}$ the charge and mass of the species $j, \phi \approx \phi_{0}(\psi)$ the electrostatic potential, and $\psi$ the toroidal flux function. Parallel and perpendicular refer to the direction of the local magnetic field, $\boldsymbol{v}_{d j}$ denotes the sum of the magnetic and $\boldsymbol{E} \times \boldsymbol{B}$ drifts, $C_{j}$ is the FokkerPlanck collision operator, and $f_{j 0}$ is the lowest order distribution function, which is a stationary Maxwellian,

$$
f_{j 0}=\eta_{j 0}(\psi)\left(\frac{m_{j}}{2 \pi T_{j 0}(\psi)}\right)^{3 / 2} \exp \left(-\frac{m_{j} \epsilon}{T_{j 0}(\psi)}\right),
$$

with $n_{j 0}=\eta_{j 0}(\psi) \exp \left(-e_{j} \phi / T_{j 0}\right)$ and $T_{j 0}$ the lowest order density and temperature of species $j$. Multiplying (1) by $T_{j 0}(\psi) f_{j 1} / f_{j 0}$, integrating over velocity space, and fluxsurface averaging $\langle\ldots\rangle$ gives the entropy balance equation for species $j$. Summing all such equations and assuming small radial density and temperature gradients gives an expression for the radial plasma current:

$$
\phi_{0}^{\prime}(\psi)\langle\boldsymbol{J} \cdot \nabla \psi\rangle=\sum_{j} T_{j 0}\left\langle\int \mathrm{d}^{3} v f_{j 1} C_{j}\left(f_{j 1}\right) / f_{j 0}\right\rangle \leq 0 .
$$

The radial transport is intrinsically ambipolar if and only if $\langle\boldsymbol{J} \cdot \boldsymbol{\nabla} \psi\rangle=0$ for an arbitrary $\phi_{0}^{\prime}(\psi)$. The H-theorem then implies

$$
f_{j 1}=\left(\alpha_{j}+\beta_{j} v_{\|}+\gamma_{j} v^{2} / 2\right) f_{j 0}
$$

Substituting this expression in (1) gives $\alpha_{j}=\gamma_{j}=0, \beta_{j}=F_{1}(\psi) / B$, and

$$
\boldsymbol{B} \times \boldsymbol{\nabla} B \cdot \boldsymbol{\nabla} \psi=F(\psi) \boldsymbol{B} \cdot \boldsymbol{\nabla} B
$$


with $F$ and $F_{1}$ arbitrary flux functions. It is easy to show that (5) is equivalent to the quasisymmetry condition $B=B\left(\psi, m \theta_{B}-n \varphi_{B}\right)$, so that the radial transport is intrinsically ambipolar and therefore plasma rotation is unconstrained if [6] and only if [9] the toroidal magnetic field is quasisymmetric. The quasisymmetry constraint (5) is clearly satisfied by the axisymmetric tokamak magnetic field,

$$
\boldsymbol{B}=I(\psi) \nabla \zeta+\nabla \zeta \times \nabla \psi
$$

with $\zeta$ the negative of the ordinary cylindrical angle measured about the toroidal axis, giving $F(\psi)=-I(\psi)$.

References $[9,12]$ further show that neither electrostatic nor electromagnetic gyrokinetic turbulence can appreciably affect the large-scale radial electric field and plasma rotation in a generic stellarator, which are therefore close to the values obtained from neoclassical theory, see also [14]. In such configurations, the turbulence effects on plasma rotation can only be important on radial scales comparable to the ion gyroradius. Only in a magnetic configuration that is very close to being perfectly quasisymmetric the bulk plasma rotation can be significantly affected by gyrokinetic turbulence.

\section{EQUILIBRIUM OF A RAPIDLY ROTATING TOROIDAL PLASMA}

The possibility of rapid (e.g. sonic) plasma rotation in a given toroidal magnetic configuration is sometimes emphasized as an advantage, since it can lead to the reduction of turbulent transport via enhanced flow shear [10]. Therefore, we investigate in the following sections whether such a rotation is possible in a quasisymmetric magnetic field.

First, we follow [11] and study a large-flow equilibrium of a toroidal plasma. We employ a conservative, steady-state form of the large-flow drift kinetic equation of 
Hazeltine and Hinton [15] for ions and expand it in the small ion gyroradius by writing $f_{i}=f_{i 0}+f_{i 1}+\ldots$ This gives in lowest order

$$
\left.\boldsymbol{\nabla}\right|_{w, \mu} \cdot\left[\frac{B f_{i 0}}{v_{\|}}\left(v_{\|} \hat{\boldsymbol{b}}+\boldsymbol{V}\right)\right]+\frac{\partial}{\partial w}\left(\frac{B f_{i 0}}{v_{\|}} \dot{w}_{i 0}\right)=\frac{B}{v_{\|}} C_{i}\left(f_{i 0}\right)
$$

Here, $\hat{\boldsymbol{b}}=\boldsymbol{B} / B, c$ is the speed of light, and $\boldsymbol{V}=V_{\|} \hat{\boldsymbol{b}}+c \hat{\boldsymbol{b}} \times \nabla\langle\phi\rangle / B$ approximates the rest frame of the distribution function $f_{i}$. The velocity $\mathbf{v}=v_{\|} \hat{\boldsymbol{b}}+\mathbf{v}_{\perp}$ is measured relative to this frame, $w=v^{2} / 2$ is the corresponding kinetic energy and $\mu=v_{\perp}^{2} / 2 B$ the magnetic moment. Furthermore

$$
\dot{w}_{i 0}=v_{\|} F_{\| i}+\mu B \boldsymbol{V} \cdot \nabla \ln B-v_{\|}^{2} \hat{\boldsymbol{b}} \cdot \nabla \boldsymbol{V} \cdot \hat{\boldsymbol{b}}
$$

where

$$
\boldsymbol{F}_{i}=-\frac{e}{m_{i}} \boldsymbol{\nabla} \tilde{\phi}-\boldsymbol{V} \cdot \boldsymbol{\nabla} \boldsymbol{V}
$$

with $e_{i}=e$ the proton charge (we consider for simplicity a hydrogen plasma) and $\tilde{\phi} \equiv \phi-\langle\phi\rangle$ the flux-surface variation of the electrostatic potential.

Equation (7) was solved in [11] to find that $f_{i 0}$ is a Maxwellian (see also [16]),

$$
f_{i 0}=\eta_{i 0}(\psi)\left(\frac{m_{i}}{2 \pi T_{i 0}(\psi)}\right)^{3 / 2} \exp \left(-\frac{2 m_{i} w+2 e \tilde{\phi}-m_{i} V^{2}}{2 T_{i 0}(\psi)}\right),
$$

and that the rapid flow $\boldsymbol{V}$, which is required to be incompressible, is given by

$$
\boldsymbol{V}=-c \frac{\boldsymbol{\nabla}\langle\phi\rangle \times \nabla B}{\boldsymbol{B} \cdot \boldsymbol{\nabla} B}
$$

When applied to this expression, the flow incompressibility requirement, $\boldsymbol{\nabla} \cdot \boldsymbol{V}=0$, constrains $\boldsymbol{B}$ to satisfy

$$
(\nabla \psi \times \nabla B) \cdot \nabla(B \cdot \nabla B)=0 .
$$

Appendix A demonstrates that constraint (12) on $\boldsymbol{B}$ is equivalent to the quasisymmetry criterion (5). Consequently, a toroidal magnetically confined plasma can only 
rotate rapidly if $\boldsymbol{B}$ is quasisymmetric. The condition (12) also implies that the magnetic field strength only depends on the flux surface and the arc length along the field, $B=f(\psi, \ell) \quad[11]$.

It is instructive to see that the flow velocity (11) reduces to the standard axisymmetric tokamak result $[16,17]$ when $\boldsymbol{B}$ is given by (6). Indeed, in this case, the magnetic field magnitude is independent of $\zeta$, i.e., $B=B(\psi, \theta)$, with $\theta$ the poloidal angle, so that $\boldsymbol{\nabla} B=(\partial B / \partial \psi) \boldsymbol{\nabla} \psi+(\partial B / \partial \theta) \boldsymbol{\nabla} \theta$. Then, using this expression in (11) and employing (6) to show $\boldsymbol{\nabla} \psi=\boldsymbol{B} \times R^{2} \boldsymbol{\nabla} \zeta$, so that $\boldsymbol{\nabla} \psi \times \boldsymbol{\nabla} \theta=(\boldsymbol{B} \cdot \boldsymbol{\nabla} \theta) R^{2} \boldsymbol{\nabla} \zeta$, gives

$$
\boldsymbol{V}=-c \frac{\mathrm{d}\langle\phi\rangle}{\mathrm{d} \psi} R^{2} \nabla \zeta
$$

as expected.

Finally, it is shown in Appendix B that

$$
\tilde{\phi}=\frac{T_{e 0}(\psi)}{2 e} \frac{m_{i}\left(V^{2}-\left\langle V^{2}\right\rangle\right)}{T_{e 0}(\psi)+T_{i 0}(\psi)}
$$

with $T_{e 0}(\psi)$ the lowest-order electron temperature.

\section{NON-AMBIPOLAR TRANSPORT IN A RAPIDLY ROTATING QUASISYMMETRIC PLASMA}

Although the lowest-order ion drift-kinetic equation (7) predicts that rapid (e.g. sonic) plasma rotation is allowed both in a quasisymmetric toroidal magnetic field of a stellarator and in an axisymmetric magnetic field of a tokamak, the two cases are actually different. In both cases, the centrifugal force associated with the rotation causes the density and electrostatic potential variation (14) within flux surfaces. However, while axisymmetry is preserved when these variations are taken into account, quasisymmetry is not. This results in the reappearance, in the latter case, of 
the $1 / \nu$-regime of neoclassical transport. In this section, we explicitly evaluate the electron collisional transport caused by this effect.

We will employ the flux coordinates $\left(\psi, \alpha \equiv \theta_{B}-\iota \varphi_{B}, \theta_{B}\right)$, with $\iota(\psi)$ the rotational transform, which derive from the usual Boozer coordinates $\left(\psi, \theta_{B}, \varphi_{B}\right)$. Then, the magnetic field can be expressed as

$$
\boldsymbol{B}=\beta_{B} \boldsymbol{\nabla} \psi+I(\psi) \boldsymbol{\nabla} \theta_{B}+J(\psi) \boldsymbol{\nabla} \varphi_{B}=\boldsymbol{\nabla} \psi \times \boldsymbol{\nabla} \alpha
$$

The Jacobian can be conveniently written as $(\nabla \psi \times \nabla \alpha) \cdot \nabla \theta_{B}=\iota B^{2} /(\iota I+J)$. For simplicity we take $\boldsymbol{B}$ to be quasiaxisymmetric, i.e., $B=B\left(\psi, \theta_{B}\right)$, but an entirely analogous calculation holds for quasi-helically symmetric fields.

Strictly speaking, Boozer coordinates are defined in the usual way only for equilibria satisfying $c^{-1} \boldsymbol{J} \times \boldsymbol{B}=\boldsymbol{\nabla} p$, which is not the case for rotating plasmas. When the pressure is anisotropic, the coordinates must be modified [18]. In the present case, the modifications would be of order $M^{2} \beta$, and will be neglected since we will eventually take $M^{2} \ll 1$ and the plasma $\beta$, which is the ratio of kinetic to magnetic pressures, is usually much smaller than unity in stellarators.

We start from the electron version of the standard drift-kinetic equation (1), with $f_{e 0}$ given by (2), where we retain the flux-surface varying portion of the electrostatic potential, $\tilde{\phi}$. This equation is appropriate for electrons even for sonic plasma flows since such flows are still much slower than the electron thermal speed as long as $M \ll\left(m_{i} / m_{e}\right)^{1 / 2}$. To solve (1) analytically we employ an additional small parameter, the ratio of the characteristic parallel length scale to the electron mean-free path, and perform an auxiliary expansion of the electron distribution function in this ratio: $f_{e 1}=f_{e 1}^{(0)}+f_{e 1}^{(1)}+\ldots$. Then, the lowest-order form of (1) becomes

$$
\nabla_{\|} f_{e 1}^{(0)}=0
$$

so that $f_{e 1}^{(0)}=f_{e 1}^{(0)}(\psi, \epsilon, \mu)$ for passing electrons and $f_{e 1}^{(0)}=f_{e 1}^{(0)}(\psi, \alpha, \epsilon, \mu)$ for trapped 
ones. The function $f_{e 1}^{(0)}$ is obtained from the next-order form of (1),

$$
v_{\|} \nabla_{\|} f_{e 1}^{(1)}+\boldsymbol{v}_{d e} \cdot \nabla f_{e 0}=C_{e}\left(f_{e 1}^{(0)}\right)
$$

by annihilating the streaming term. Equation (17) [and (1)] neglects the term $\boldsymbol{v}_{d e} \cdot \boldsymbol{\nabla} f_{e 1}^{(0)}$ that can lead to the $\sqrt{\nu_{e}}$-regime of neoclassical transport [19]. This approximation is justified whenever $M$ is small compared with $\left(m_{i} / m_{e}\right)^{1 / 2}$ times the ratio of the characteristic length scale along the $\alpha$-direction to the (effective) electron mean-free path.

As usual, the annihilation operator is the flux-surface average $\left\langle B / v_{\|}\right\rangle^{-1}\left\langle\left(B / v_{\|}\right)(\cdots)\right\rangle$ for passing electrons and the bounce average $\overline{(\cdots)} \equiv \tau_{B}^{-1} \oint\left(\mathrm{d} \ell / v_{\|}\right)(\cdots)$ for trapped ones, where $\ell$ is the arc length along the magnetic field, $\tau_{B} \equiv \oint\left(\mathrm{d} \ell / v_{\|}\right)$is the bounce time, and the integration is performed between the bounce points. Since trapped particles are responsible for the $1 / \nu$-regime of transport, we concentrate on evaluating $f_{e 1}^{(0)}$ only for such particles. Then, (17) becomes

$$
\overline{\boldsymbol{v}_{d e} \cdot \boldsymbol{\nabla} \psi} \frac{\partial f_{e 0}}{\partial \psi}=\overline{C_{e}\left(f_{e 1}^{(0)}\right)}
$$

To proceed we need to evaluate bounce averages of the electron radial drift velocity and collision operator. Since $\boldsymbol{v}_{d e}=\left(v_{\|} / B\right) \boldsymbol{\nabla} \times\left(v_{\|} \boldsymbol{B} / \Omega_{e}\right)$, with $\Omega_{e} \equiv-e B /\left(m_{e} c\right)$ the electron gyrofrequency, we have

$$
\boldsymbol{v}_{d e} \cdot \boldsymbol{\nabla} \psi=\frac{v_{\|}}{\Omega_{e}} \boldsymbol{\nabla} \cdot\left(v_{\|} \hat{\boldsymbol{b}} \times \boldsymbol{\nabla} \psi\right)=\frac{v_{\|} B^{2}}{\Omega_{e}(\iota I+J)}\left[(\iota I+J) \frac{\partial}{\partial \alpha}+J \frac{\partial}{\partial \theta_{B}}\right]\left(\frac{v_{\|}}{B}\right) .
$$

Moreover,

$$
\boldsymbol{B} \cdot \boldsymbol{\nabla} B=B \frac{\partial B}{\partial \ell}=(\boldsymbol{\nabla} \psi \times \boldsymbol{\nabla} \alpha) \cdot \boldsymbol{\nabla} \theta_{B} \frac{\partial B}{\partial \theta_{B}}=\frac{\iota B^{2}}{\iota I+J} \frac{\partial B}{\partial \theta_{B}}
$$

so that

$$
\mathrm{d} \ell=\frac{\iota I+J}{\iota B} \mathrm{~d} \theta_{B} .
$$


Consequently,

$$
\overline{\boldsymbol{v}_{d e} \cdot \boldsymbol{\nabla} \psi}=\frac{(\iota I+J) B}{\iota \tau_{B} \Omega_{e}} \oint \mathrm{d} \theta_{B} \frac{\partial}{\partial \alpha}\left(\frac{v_{\|}}{B}\right) .
$$

Expressing $v_{\|}$in terms of $\varepsilon, \mu$, and $\phi$, recalling that $B=B\left(\psi, \theta_{B}\right)$, and evaluating

$$
\frac{\partial}{\partial \alpha}\left(\frac{v_{\|}}{B}\right)=\frac{e}{m_{e} B v_{\|}} \frac{\partial \tilde{\phi}}{\partial \alpha}
$$

finally results in

$$
\overline{\boldsymbol{v}_{d e} \cdot \nabla \psi}=-c \frac{\iota I+J}{\iota \tau_{B}} \oint \frac{\mathrm{d} \theta_{B}}{v_{\|} B} \frac{\partial \tilde{\phi}}{\partial \alpha} .
$$

Next, we evaluate the bounce average of the electron collision operator $C_{e}\left(f_{e 1}^{(0)}\right)=$ $C_{e e}\left(f_{e 1}^{(0)}\right)+C_{e i}\left(f_{e 1}^{(0)}\right)$. We employ the usual model for the electron-ion operator [8]:

$$
C_{e i}\left(f_{e 1}^{(0)}\right)=\nu_{e i}(v)\left[\mathcal{L}\left(f_{e 1}^{(0)}\right)+\frac{m_{e} v_{\|} V_{\| i}}{T_{e 0}} f_{e 0}\right]
$$

Here, $\mathcal{L}$ is the Lorentz operator, $V_{\| i}$ is the ion parallel flow velocity, and the velocitydependent electron-ion collision frequency is expressed in terms of the usual electron collision frequency [20],

$$
\nu_{e} \equiv \frac{4(2 \pi)^{1 / 2} n_{e 0} e^{4} \Lambda}{3 m_{e}^{1 / 2} T_{e 0}^{3 / 2}},
$$

as

$$
\nu_{e i}(v) \equiv \frac{3 \pi^{1 / 2}}{4} \nu_{e}\left(\frac{v_{T e}}{v}\right)^{3}
$$

with $\Lambda$ the Coulomb logarithm and $v_{T e} \equiv \sqrt{2 T_{e 0} / m_{e}}$ the electron thermal speed. We also use a similar simple model for the electron-electron collision operator [8],

$$
C_{e e}\left(f_{e 1}^{(0)}\right)=\nu_{e e}(v)\left[\mathcal{L}\left(f_{e 1}^{(0)}\right)+\frac{m_{e} v_{\|} u_{\|}}{T_{e 0}} f_{e 0}\right],
$$

where $u_{\|}$is chosen to make the $C_{e e}$ conserve momentum and is not required for the present calculation, and

$$
\nu_{e e}(v) \equiv \nu_{e i}(v)\left[\left(1-\frac{1}{2 x^{2}}\right) \operatorname{erf}(x)+\frac{1}{2 x} \frac{\mathrm{d} \operatorname{erf}(x)}{\mathrm{d} x}\right]
$$


with $\operatorname{erf}(x) \equiv(2 / \sqrt{\pi}) \int_{0}^{x} \mathrm{~d} t \exp \left(-t^{2}\right)$ the error function and $x \equiv v / v_{T e}$. Since the terms proportional to $v_{\|} f_{e 0}$ do not contribute to the bounce average of $C_{e}$, we obtain

$$
\overline{C_{e}\left(f_{e 1}^{(0)}\right)}=\frac{1}{\tau_{B}} \oint \frac{\mathrm{d} \ell}{v_{\|}} \nu_{e}(v) \mathcal{L}\left(f_{e 1}^{(0)}\right)=\frac{\iota I+J}{\iota \tau_{B}} \oint \frac{\mathrm{d} \theta_{B}}{v_{\|} B} \nu_{e}(v) \mathcal{L}\left(f_{e 1}^{(0)}\right)
$$

with $\nu_{D}(v) \equiv \nu_{e e}(v)+\nu_{e i}(v)$. It is convenient to express the Lorentz operator in terms of the variables $\xi \equiv v_{\|} / v$ and $\lambda \equiv \mu / w=v_{\perp}^{2} /\left(v^{2} B\right)$, so that

$$
\mathcal{L}\left(f_{e 1}^{(0)}\right)=\frac{2 \xi}{B} \frac{\partial}{\partial \lambda}\left(\lambda \xi \frac{\partial f_{e 1}^{(0)}}{\partial \lambda}\right)
$$

and

$$
\overline{C_{e}\left(f_{e 1}^{(0)}\right)}=\frac{\iota I+J}{\iota \tau_{B}} \oint \mathrm{d} \theta_{B} \frac{2 \nu_{D}(v)}{v B^{2}} \frac{\partial}{\partial \lambda}\left(\lambda \xi \frac{\partial f_{e 1}^{(0)}}{\partial \lambda}\right) .
$$

To simplify (32) further we recall that the total electron energy does not vary along its trajectory, $\Delta \varepsilon=0$, so that the relative variation in the electron velocity along the trajectory can be expressed in terms of the flux-surface variation of the electrostatic potential as $\Delta v / v \sim e \tilde{\phi} / T_{e 0}$. Employing (14) then gives

$$
\frac{\Delta v}{v} \sim \frac{m_{i}\left(V^{2}-\left\langle V^{2}\right\rangle\right)}{T_{e 0}+T_{i 0}} \sim M^{2}-\left\langle M^{2}\right\rangle
$$

where $M^{2} \equiv m_{i} V^{2} /\left(T_{e 0}+T_{i 0}\right)$ is the plasma flow Mach number. Next, using (11) to write

$$
V^{2}-\left\langle V^{2}\right\rangle=\left[c\langle\phi\rangle^{\prime}\left(\frac{\iota I+J}{\iota}\right)\right]^{2}\left(\frac{B_{T}^{2}}{B^{4}}-\left\langle\frac{B_{T}^{2}}{B^{4}}\right\rangle\right),
$$

with $\boldsymbol{B}_{T} \equiv \boldsymbol{\nabla} \psi \times \boldsymbol{\nabla} \theta_{B}$ the toroidal magnetic field, assuming $B_{T} \approx B$, and also assuming that the relative variation of the magnetic field along the electron trajectory is small, $\Delta B / B \ll 1$, results in the final estimate

$$
\frac{\Delta v}{v} \sim \frac{e \tilde{\phi}}{T_{e 0}} \sim M^{2} \frac{\Delta B}{B}
$$

Similarly, the variation in $\xi$ along the trajectory is of order

$$
\frac{\Delta \xi}{\xi} \sim \frac{\Delta v}{v}+\frac{1}{\xi^{2}}\left(\frac{\Delta B}{B}-\frac{e \tilde{\phi}}{T_{e 0}}\right),
$$


and is, by definition, of order unity for trapped particles. Consequently, for $M^{2} \ll 1$ we can neglect variations in $v$ and $\lambda, \Delta \lambda / \lambda \sim \Delta v / v$, but retain variations in $\xi$ and $B$, along the electron trajectory when evaluating the integral in (32). As a result, (32) becomes approximately

$$
\overline{C_{e}\left(f_{e 1}^{(0)}\right)} \approx \frac{\iota I+J}{\iota \tau_{B}} \frac{2 \nu_{D}(v)}{v} \frac{\partial}{\partial \lambda}\left[\lambda \frac{\partial f_{e 1}^{(0)}}{\partial \lambda}\left(\oint \mathrm{d} \theta_{B} \frac{\sqrt{1-\lambda B}}{B^{2}}\right)\right] .
$$

Performing similar manipulations with (24) and using the results in (18) gives the final equation for $f_{e 1}^{(0)}$ :

$$
2 \nu_{D}(v) \frac{\partial}{\partial \lambda}\left[\lambda \frac{\partial f_{e 1}^{(0)}}{\partial \lambda}\left(\oint \mathrm{d} \theta_{B} \frac{\sqrt{1-\lambda B}}{B^{2}}\right)\right]=-c \frac{\partial f_{e 0}}{\partial \psi} \oint \frac{\partial \tilde{\phi}}{\partial \alpha} \frac{\mathrm{d} \theta_{B}}{B \sqrt{1-\lambda B}} .
$$

Integrating this equation once gives

$$
\frac{\partial f_{e 1}^{(0)}}{\partial \lambda}=\frac{c}{2 \nu_{D}(v)} \frac{\partial f_{e 0}}{\partial \psi} \frac{1}{\lambda \oint \mathrm{d} \theta_{B} B^{-2} \sqrt{1-\lambda B}} \int_{\lambda}^{1 / B_{\min }} \mathrm{d} \lambda_{1} \oint \frac{\partial \tilde{\phi}}{\partial \alpha} \frac{\mathrm{d} \theta_{B}}{B \sqrt{1-\lambda_{1} B}},
$$

where $B_{\min }$ denotes the local minimum of the magnetic field magnitude on a given field line. Since $f_{e 1}^{(0)}$ is independent of $\alpha$ for passing particles, but dependent on $\alpha$ for trapped particles, it should vanish at the trapped-passing boundary $\lambda=1 / B_{\text {max }}$, where $B_{\max }$ denotes the maximum of the magnetic field magnitude on the flux surface. Employing this boundary condition and integrating (39) gives the final expression for the trapped-particle distribution $f_{e 1}^{(0)}$ :

$$
f_{e 1}^{(0)}=\frac{c}{2 \nu_{D}(v)} \frac{\partial f_{e 0}}{\partial \psi} \int_{1 / B_{\max }}^{\lambda} \frac{\mathrm{d} \lambda_{1}}{\lambda_{1} \oint \mathrm{d} \theta_{B} B^{-2} \sqrt{1-\lambda_{1} B}} \int_{\lambda_{1}}^{1 / B_{\min }} \mathrm{d} \lambda_{2} \oint \frac{\partial \tilde{\phi}}{\partial \alpha} \frac{\mathrm{d} \theta_{B}}{B \sqrt{1-\lambda_{2} B}} .
$$

Next, we evaluate the radial particle flux due to the trapped electrons. Starting from the standard expression

$$
\left\langle\boldsymbol{\Gamma}_{e} \cdot \nabla \psi\right\rangle \equiv\left\langle\int \mathrm{d}^{3} v\left(\boldsymbol{v}_{d e} \cdot \nabla \psi\right) f_{e 1}^{(0)}\right\rangle,
$$

employing (19) for $\boldsymbol{v}_{d e} \cdot \boldsymbol{\nabla} \psi$, and recalling that $f_{e 1}^{(0)}$ is independent of $\theta_{B}$ gives

$$
\left\langle\boldsymbol{\Gamma}_{e} \cdot \nabla \psi\right\rangle=\left\langle\int \mathrm{d}^{3} v \frac{v_{\|} B^{2}}{\Omega_{e}} \frac{\partial}{\partial \alpha}\left(\frac{v_{\|}}{B}\right) f_{e 1}^{(0)}\right\rangle .
$$


Employing (23) results in

$$
\begin{array}{r}
\left\langle\boldsymbol{\Gamma}_{e} \cdot \nabla \psi\right\rangle=-c\left\langle\frac{\partial \tilde{\phi}}{\partial \alpha} \int \mathrm{d}^{3} v f_{e 1}^{(0)}\right\rangle \\
=-c \sum_{\sigma}\left\langle\frac{\partial \tilde{\phi}}{\partial \alpha} \int_{0}^{+\infty} \mathrm{d} v \pi v^{2} \int_{0}^{1 / B} \mathrm{~d} \lambda \frac{B}{\sqrt{1-\lambda B}} f_{e 1}^{(0)}\right\rangle,
\end{array}
$$

where $\sigma$ denotes the sign of $v_{\|}$. Finally, substituting $f_{e 1}^{(0)}$ from (40) gives

$$
\begin{gathered}
\left\langle\boldsymbol{\Gamma}_{e} \cdot \nabla \psi\right\rangle=-c^{2}\left\langle B \frac{\partial \tilde{\phi}}{\partial \alpha} \int_{0}^{+\infty} \mathrm{d} v \frac{\pi v^{2}}{\nu_{D}(v)} \frac{\partial f_{e 0}}{\partial \psi} \int_{1 / B_{\max }}^{1 / B} \frac{\mathrm{d} \lambda}{\sqrt{1-\lambda B}} \times\right. \\
\left.\int_{1 / B_{\max }}^{\lambda} \frac{\mathrm{d} \lambda_{1}}{\lambda_{1} \oint \mathrm{d} \theta_{B} B^{-2} \sqrt{1-\lambda_{1} B}} \int_{\lambda_{1}}^{1 / B_{\min }} \mathrm{d} \lambda_{2} \oint \frac{\partial \tilde{\phi}}{\partial \alpha} \frac{\mathrm{d} \theta_{B}}{B \sqrt{1-\lambda_{2} B}}\right\rangle .
\end{gathered}
$$

The integral in $v$ can be easily evaluated by summing (27) and (29) to obtain $\nu_{D}(v)$ and using (2) for $f_{e 0}$ :

$$
\int_{0}^{+\infty} \mathrm{d} v \frac{\pi v^{2}}{\nu_{D}(v)} \frac{\partial f_{e 0}}{\partial \psi}=\frac{n_{e 0}}{\nu_{e}}\left[0.237\left(\frac{\mathrm{d} \ln n_{e 0}}{\mathrm{~d} \psi}-\frac{e}{T_{e 0}} \frac{\mathrm{d} \phi_{0}}{\mathrm{~d} \psi}\right)+0.335 \frac{\mathrm{d} \ln T_{e 0}}{\mathrm{~d} \psi}\right] .
$$

The only remaining quantity required for evaluating the $\lambda$-integrals in (44) is $\partial \tilde{\phi} / \partial \alpha$, which can be obtained from (14) and (34):

$$
\begin{array}{r}
\frac{\partial \tilde{\phi}}{\partial \alpha}=\frac{m_{i} T_{e 0}}{2 e\left(T_{e 0}+T_{i 0}\right)} \frac{\partial V^{2}}{\partial \alpha}=\frac{m_{i} T_{e 0}}{2 e\left(T_{e 0}+T_{i 0}\right)}\left[\frac{c\langle\phi\rangle^{\prime}}{B^{2}}\left(\frac{\iota I+J}{\iota}\right)\right]^{2} \frac{\partial B_{T}^{2}}{\partial \alpha} \\
\propto M^{2} \frac{1}{B^{2}} \frac{\partial\left(B^{2}-B_{T}^{2}\right)}{\partial \alpha},
\end{array}
$$

where we recalled that $B=B\left(\psi, \theta_{B}\right)$. Therefore, it is clear that

$$
\left\langle\boldsymbol{\Gamma}_{e} \cdot \nabla \psi\right\rangle \propto \frac{M^{4}}{\nu_{e}}\left[\frac{1}{B^{2}} \frac{\partial\left(B^{2}-B_{T}^{2}\right)}{\partial \alpha}\right]^{2}
$$

where $\left(B^{2}-B_{T}^{2}\right) / B^{2} \ll 1$

Expression (44) can be simplified if we assume that $B=B_{0}\left(1-\epsilon \cos \theta_{B}\right)$, with $\epsilon \ll 1$, and change variables from $\lambda$ to $\kappa$, which is defined as

$$
\kappa^{2} \equiv \frac{1-\lambda B_{0}(1-\epsilon)}{2 \epsilon \lambda B_{0}} \approx \frac{1-\lambda B_{0}(1-\epsilon)}{2 \epsilon}
$$


Then, $1-\lambda B \approx 2 \epsilon\left[\kappa^{2}-\sin ^{2}\left(\theta_{B} / 2\right)\right]$, and we obtain to lowest order in $\epsilon$

$$
\oint \frac{\partial \tilde{\phi}}{\partial \alpha} \frac{\mathrm{d} \theta_{B}}{B \sqrt{1-\lambda_{2} B}}=\frac{h\left(\psi, \alpha, \kappa_{2}\right)}{\sqrt{2 \epsilon} B_{0}}
$$

with

$$
h(\psi, \alpha, \kappa) \equiv \oint \frac{\partial \tilde{\phi}}{\partial \alpha} \frac{\mathrm{d} \theta_{B}}{\sqrt{\kappa^{2}-\sin ^{2}\left(\theta_{B} / 2\right)}}
$$

Similarly,

$$
\begin{array}{r}
\int_{\lambda_{1}}^{1 / B_{\min }} \mathrm{d} \lambda_{2}=\frac{4 \epsilon}{B_{0}} \int_{0}^{\kappa_{1}} \mathrm{~d} \kappa_{2} \kappa_{2}, \\
\int_{1 / B_{\max }}^{\lambda} \frac{\mathrm{d} \lambda_{1}}{\lambda_{1}}=4 \epsilon \int_{\kappa}^{1} \mathrm{~d} \kappa_{1} \kappa_{1}, \\
\oint \mathrm{d} \theta_{B} \frac{\sqrt{1-\lambda_{1} B}}{B^{2}}=\frac{8 \sqrt{2 \epsilon}}{B_{0}^{2}}\left[\mathrm{E}\left(\kappa_{1}\right)+\left(\kappa_{1}^{2}-1\right) \mathrm{K}\left(\kappa_{1}\right)\right], \\
\oint \frac{\mathrm{d} \theta_{B}}{B^{2}} B \frac{\partial \tilde{\phi}}{\partial \alpha} \int_{1 / B_{\max }}^{1 / B} \frac{\mathrm{d} \lambda}{\sqrt{1-\lambda B}}=\int_{1 / B_{\max }}^{1 / B_{\min }} \mathrm{d} \lambda \oint \frac{\partial \tilde{\phi}}{\partial \alpha} \frac{\mathrm{d} \theta_{B}}{B \sqrt{1-\lambda B}} \\
=\frac{2 \sqrt{2 \epsilon}}{B_{0}^{2}} \int_{0}^{1} \mathrm{~d} \kappa \kappa h(\psi, \alpha, \kappa),
\end{array}
$$

with $\mathrm{E}$ and $\mathrm{K}$ the complete elliptical integrals of the first and second kind, respectively. Employing these results in (44) gives the following simplified expression for the electron radial flux:

$$
\begin{gathered}
\left\langle\boldsymbol{\Gamma}_{e} \cdot \nabla \psi\right\rangle=-\frac{c^{2} n_{e 0}(2 \epsilon)^{3 / 2}}{4 \pi^{2} \nu_{e} \iota}\left[0.237\left(\frac{\mathrm{d} \ln n_{e 0}}{\mathrm{~d} \psi}-\frac{e}{T_{e 0}} \frac{\mathrm{d} \phi_{0}}{\mathrm{~d} \psi}\right)+0.335 \frac{\mathrm{d} \ln T_{e 0}}{\mathrm{~d} \psi}\right] \times \\
\int_{0}^{2 \pi \iota} \mathrm{d} \alpha \int_{0}^{1} \mathrm{~d} \kappa \kappa h(\psi, \alpha, \kappa) \int_{\kappa}^{1} \frac{\mathrm{d} \kappa_{1} \kappa_{1}}{\mathrm{E}\left(\kappa_{1}\right)+\left(\kappa_{1}^{2}-1\right) \mathrm{K}\left(\kappa_{1}\right)} \int_{0}^{\kappa_{1}} \mathrm{~d} \kappa_{2} \kappa_{2} h\left(\psi, \alpha, \kappa_{2}\right) .
\end{gathered}
$$

We expect in this case that $\left(1 / B^{2}\right) \partial\left(B^{2}-B_{T}^{2}\right) / \partial \alpha \approx\left(2 / B^{2}\right) \partial\left(\boldsymbol{B}_{T} \cdot \boldsymbol{B}_{P}\right) / \partial \alpha \propto \epsilon$, with $\boldsymbol{B}_{P} \equiv-\iota \boldsymbol{\nabla} \psi \times \boldsymbol{\nabla} \varphi_{B}$ the poloidal magnetic field, so that $\left\langle\boldsymbol{\Gamma}_{e} \cdot \boldsymbol{\nabla} \psi\right\rangle \propto \epsilon^{7 / 2}$.

\section{CONCLUSIONS}

In general, the equilibrium large-scale plasma rotation in a toroidal magnetic field must be subsonic, and is determined by the requirement that collisional particle transport should be ambipolar to lowest order. Neither electrostatic nor electromagnetic 
gyrokinetic turbulence can appreciably modify this rotation and the associated radial electric field, except transiently or on radial length scales comparable to the ion gyroradius. Only in quasisymmetric toroidal magnetic fields is the collisional particle transport intrinsically ambipolar, so that the plasma can rotate freely in the quasisymmetry direction. Although it is very difficult to realise quasisymmetry to high enough accuracy, in principle one would think that the rotation velocity in such a device could approach the ion thermal speed. However, if it does, then the quasisymmetry gets violated and the centrifugal force becomes important in determining the particle distribution within flux surfaces. This leads to the reappearance of a $1 / \nu$ regime of neoclassical transport, which is not intrinsically ambipolar and normally absent in quasisymmetric magnetic fields. Fortunately, this transport is relatively weak. For electrons, it scales as $M^{4} / \nu_{e}$ times a small geometric factor (proportional to $\epsilon^{7 / 2}$ for large aspect-ratio, $\epsilon \ll 1$, configurations). Since $M$ and $\epsilon$ are expected to be small in most plasmas of interest, the non-ambipolar $1 / \nu$-transport is expected to be very modest.

\section{ACKNOWLEDGEMENT}

This work was partially supported by the U.S.A. Department of Energy grant DE-AC52-06NA-25396 at Los Alamos National Laboratory. A.N.S. is grateful to Max-Planck-Institut für Plasmaphysik, Greifswald, Germany for its hospitality and support during the course of this work.

\section{APPENDIX A: EQUIVALENCE OF (5) AND (12)}

This appendix demonstrates that magnetic field constraint (12) is equivalent to the quasisymmetry criterion (5), see also Ref. [21]. To see this we notice that the 
vector $\boldsymbol{B} \times \boldsymbol{\nabla} \psi$ is tangential to a flux surface and thus can be written as

$$
\boldsymbol{B} \times \boldsymbol{\nabla} \psi=a \boldsymbol{B}+b \boldsymbol{\nabla} \psi \times \boldsymbol{\nabla} B
$$

with some scalar quantities $a$ and $b$. Dotting this equation with $\nabla B$ gives $a=$ $(\boldsymbol{B} \times \boldsymbol{\nabla} \psi \cdot \boldsymbol{\nabla} B) / \boldsymbol{B} \cdot \boldsymbol{\nabla} B$, while crossing it with $\boldsymbol{B}$ gives $b=-B^{2} / \boldsymbol{B} \cdot \boldsymbol{\nabla} B$. Evaluating divergence of (A1), employing $\boldsymbol{J} \cdot \boldsymbol{\nabla} \psi \approx 0$, and using (12) results in

$$
\boldsymbol{B} \cdot \boldsymbol{\nabla} a \approx \nabla \psi \times \nabla B \cdot\left(\frac{B^{2}}{\boldsymbol{B} \cdot \boldsymbol{\nabla} B}\right)=0
$$

Therefore, $a$ is a flux function on irrational flux surfaces, and by continuity also on rational ones, which is equivalent to (5).

\section{APPENDIX B: FLUX-SURFACE VARIATION OF ELECTROSTATIC POTENTIAL}

This appendix evaluates the flux-surface variation of the electrostatic potential, $\tilde{\phi}=\phi-\langle\phi\rangle$, and obtains (14). It is shown in $[11,15,16]$ that flux-surface variations of the electron and ion densities, $n_{e}$ and $n_{i}$, and the electrostatic potential are related through the constraints

$$
\begin{array}{r}
\nabla_{\|} \ln n_{e}-\frac{e}{T_{e 0}(\psi)} \nabla_{\|} \tilde{\phi}=0 \\
\nabla_{\|} \ln n_{i}+\frac{e}{T_{i 0}(\psi)} \nabla_{\|} \tilde{\phi}+\frac{m_{i}}{T_{i 0}(\psi)} \boldsymbol{V} \cdot \nabla \boldsymbol{V} \cdot \hat{\boldsymbol{b}}=0
\end{array}
$$

arising from parallel momentum balance. Subtracting one of these equations from the other, employing quasineutrality, $n_{e}=n_{i}$, and multiplying by $B$ gives

$$
\boldsymbol{B} \cdot \boldsymbol{\nabla} \tilde{\phi}=-\frac{m_{i} T_{e 0}(\psi)}{e\left[T_{e 0}(\psi)+T_{i 0}(\psi)\right]} \boldsymbol{V} \cdot \boldsymbol{\nabla} \boldsymbol{V} \cdot \boldsymbol{B}
$$

Using

$$
\boldsymbol{\nabla} \times(\boldsymbol{V} \times \boldsymbol{B})=\boldsymbol{V}(\boldsymbol{\nabla} \cdot \boldsymbol{B})-\boldsymbol{B}(\boldsymbol{\nabla} \cdot \boldsymbol{V})+\boldsymbol{B} \cdot \boldsymbol{\nabla} \boldsymbol{V}-\boldsymbol{V} \cdot \boldsymbol{\nabla} \boldsymbol{B}
$$


with $\boldsymbol{\nabla} \cdot \boldsymbol{B}=\boldsymbol{\nabla} \cdot \boldsymbol{V}=0$ and $\boldsymbol{V} \times \boldsymbol{B}=c \boldsymbol{\nabla}\langle\phi\rangle$, results in $\boldsymbol{B} \cdot \boldsymbol{\nabla} \boldsymbol{V}=\boldsymbol{V} \cdot \boldsymbol{\nabla} \boldsymbol{B}$, so that

$\boldsymbol{V} \cdot \boldsymbol{\nabla} \boldsymbol{V} \cdot \boldsymbol{B}=\boldsymbol{V} \cdot \boldsymbol{\nabla}(\boldsymbol{V} \cdot \boldsymbol{B})-\boldsymbol{V} \cdot \boldsymbol{\nabla} \boldsymbol{B} \cdot \boldsymbol{V}=\boldsymbol{V} \cdot \boldsymbol{\nabla}(\boldsymbol{V} \cdot \boldsymbol{B})-\boldsymbol{B} \cdot \boldsymbol{\nabla}\left(V^{2} / 2\right)$.

Employing (5) in (11) to show $\boldsymbol{V} \cdot \boldsymbol{B}=F(\psi) \mathrm{d}\langle\phi\rangle / \mathrm{d} \psi$, so that $\boldsymbol{V} \cdot \boldsymbol{\nabla}(\boldsymbol{V} \cdot \boldsymbol{B}) \propto$ $\boldsymbol{V} \cdot \boldsymbol{\nabla} \psi=0$, gives

$$
\boldsymbol{V} \cdot \boldsymbol{\nabla} \boldsymbol{V} \cdot \boldsymbol{B}=-\boldsymbol{B} \cdot \boldsymbol{\nabla}\left(V^{2} / 2\right)
$$

Using this result in (B2) finally gives (14).

[1] Nührenberg J, Lotz W and Gori S 1994 Theory of Fusion Plasmas (Bologna: Editrice Compositori) p 3

[2] Nührenberg J and Zille R 1988 Phys. Lett. A 129113

[3] Boozer A H 1995 Plasma Phys. Control. Fusion 37 A103

[4] Garren D A and Boozer A H 1991 Phys. Fluids B 32822

[5] Helander P and Nührenberg J 2009 Plasma Phys. Contr. Fusion 51055004

[6] Boozer A H 1983 Phys. Fluids 26496

[7] Rutherford P H 1970 Phys. Fluids 13482

[8] Helander P and Sigmar D J 2002 Collisional Transport in Magnetized Plasmas (Cambridge: Cambridge University Press)

[9] Helander P and Simakov A N 2008 Phys. Rev. Lett. 101145003

[10] Spong D A 2005 Phys. Plasmas 12056114

[11] Helander P 2007 Phys. Plasmas 14104501

[12] Helander P and Simakov A N 2010 "Gyrokinetic theory of rotation in stellarators" to appear in Contrib. Plasma Phys.

[13] Hazeltine R D 1973 Plasma Phys 1577

[14] Sugama H, Okamoto M and Horton W 1996 Phys. Plasmas 32379 
[15] Hazeltine R D and Hinton F L 2005 Phys. Plasmas 12102506

[16] Hinton F L and Wong S K 1985 Phys. Fluids 283082

[17] Catto P J, Bernstein I B and Tessarotto M 1987 Phys. Fluids 302784

[18] Cooper W A, Graves J P, Jucker M and Isaev M Yu 2006 Phys. Plasmas 13092501

[19] Ho D D-M and Kulsrud R M 1987 Phys. Fluids 30442

[20] Braginskii S I 1965 Transport processes in a plasma Reviews of Plasma Physics vol 1 ed M A Leontovich (New York: Consultants Bureau) p 205

[21] Skovoroda A A and Shafranov V D 1995 Plasma Phys. Rep. 21886 